\title{
Lenalidomide maintenance post-transplantation in newly diagnosed multiple myeloma: real-world outcomes and costs
}

\author{
Parameswaran Hari*,1, Brian Ung ${ }^{2}$, Safiya Abouzaid ${ }^{2}$, Amit Agarwal $^{2}$ \& Kejal Parikh ${ }^{2}$ \\ ${ }^{1}$ Division of Hematology \& Oncology, Medical College of Wisconsin, Milwaukee, WI 53226, USA \\ ${ }^{2}$ Celgene Corporation, Summit, NJ 07901, USA \\ *Author for correspondence: Tel.: +414 805 6800; phari@mcw.edu
}

\begin{abstract}
Aim: To compare real-world outcomes and costs among patients with newly diagnosed multiple myeloma receiving lenalidomide-only maintenance (Len-Mt) versus no maintenance after autologous hematopoietic stem cell transplantation. Patients \& methods: Time to next treatment (TTNT) was evaluated; costs were calculated for $0-12,12-24$ and 24-36 months postindex date. Results: Len-Mt cohort had longer TTNT (HR: 0.43; $p<0.0001$ ). Per-patient per-month costs during months $0-12$ were higher among patients, receiving Len-Mt (USD 13,095 vs USD 8910; $p<0.0001$ ), due to higher pharmacy costs - outpatient costs were lower. During months 12-24 and 24-36, outpatient costs were similar in both cohorts; total and pharmacy costs remained elevated for patients receiving Len-Mt. Conclusion: Len-Mt improved TTNT, initially reduced outpatient costs, but resulted in higher overall and pharmacy costs.
\end{abstract}

First draft submitted: 19 July 2019; Accepted for publication: 1 October 2019; Published online: 18 October 2019

Keywords: ASCT • autologous hematopoietic stem cell transplantation • healthcare resource utilization • lenalidomide $\bullet$ maintenance therapy $\bullet$ multiple myeloma $\bullet$ real world

The estimated annual incidence of multiple myeloma (MM) in the USA is approximately 32,110 - and has increased over the last 10 years [1,2]. Despite this increase, evidence from randomized controlled trials (RCTs) suggests that survival outcomes have improved in recent years with the availability of effective novel therapies (e.g., lenalidomide, an immune-modifying drug and bortezomib, a proteasome inhibitor) [3].

National Comprehensive Cancer Network [4] and European Society for Medical Oncology [5] guidelines recommend primary treatment options for patients with newly diagnosed MM (NDMM) that include various triplet regimens (e.g., lenalidomide/bortezomib/dexamethasone). These are commonly followed by autologous hematopoietic stem cell transplantation (ASCT) in patients eligible for transplant and maintenance therapy with lenalidomide or bortezomib $[4,5]$.

In 2017, lenalidomide became the first and currently is the only post-ASCT maintenance therapy approved in the USA [6] and Europe [7]. Approval from the US FDA was based on data from two Phase III trials (Intergroupe Francophone du Myélome [IFM] 2205-02 and Cancer and Leukemia Group B [CALGB] 100104) that showed significant improvements in progression-free survival (PFS), event-free survival and overall survival (OS) with lenalidomide maintenance compared with placebo [8,9]. In CALGB 100104, lenalidomide maintenance therapy after ASCT significantly improved the time to disease progression compared with placebo (hazard ratio [HR]: 0.57; 95\% CI: 0.46-0.71; p < 0.0001) [10]. In a crossover-adjusted analysis, a survival benefit of $>3$ years was observed [11]. Results from the Myeloma XI trial have also confirmed the significant PFS benefit of lenalidomide maintenance versus observation after ASCT [12]. A meta-analysis based on data from IFM 2205-02, CALGB 100104 and the GIMEMA RV-MM-PR-209 trial [13] compared lenalidomide maintenance versus placebo/observation after ASCT and reported significant improvements in PFS (52.8 vs 23.5 months; HR: $0.48 ; 95 \%$ CI: $0.41-0.55$ ) and OS (not reached vs 86.0 months; HR: 0.75; 95\% CI: 0.63-0.90) in patients receiving lenalidomide maintenance [14]. The survival benefit of lenalidomide maintenance post-ASCT in patients with NDMM may have direct economic

Future $\because$ Medicine 


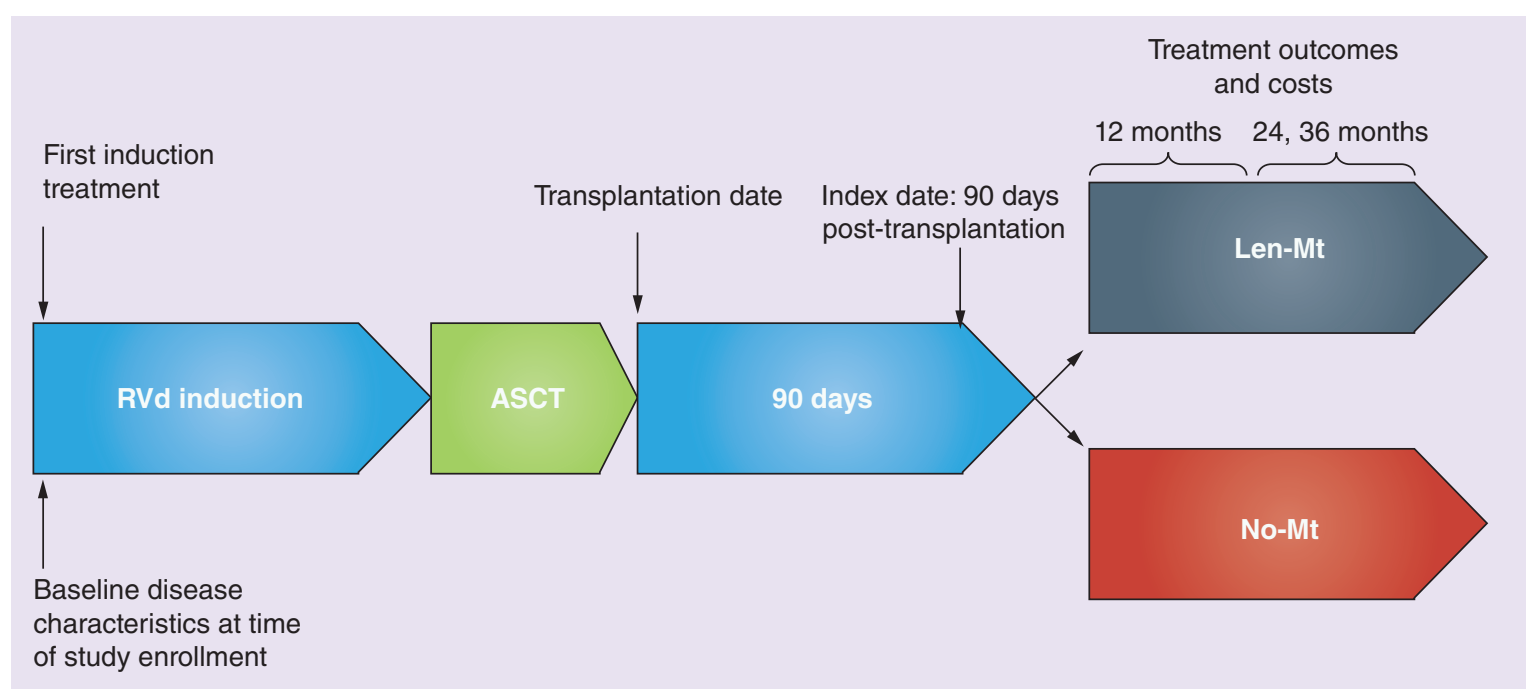

Figure 1. Study design.

ASCT: Autologous stem cell transplantation; MM: Multiple myeloma; No-Mt: No maintenance therapy; Len-Mt: Lenalidomide-only maintenance; RVd: Lenalidomide/bortezomib/dexamethasone.

consequences. For relapsed MM patients, several analyses have shown that therapies that delay disease progression and extend treatment-free intervals reduce costs and healthcare resource utilization [15-17].

Treatment patterns and costs associated with post-ASCT maintenance therapy in 'real-world' patient populations have not been well characterized for patients with NDMM. Although RCTs are needed for treatment testing and registration purposes, evaluations of care and outcomes beyond the experimental setting are likewise essential to population health management decisions. Assessment of practice patterns and understanding treatment effectiveness in patients who would not meet eligibility criteria for RCTs are critical factors for payers and other healthcare decision-makers.

Using a US claims database, this study aimed to retrospectively assess real-world treatment patterns, time to treatment discontinuation (TTTD), time to next treatment (TTNT), healthcare resource utilization and costs among patients with NDMM receiving ASCT followed by lenalidomide-only maintenance (Len-Mt) compared with those receiving no maintenance therapy.

\section{Patients \& methods}

Data source

In this retrospective observational study from the perspective of US payers, administrative claims data were obtained from the Truven MarketScan Commercial and Encounters Database (Medicare Supplemental [Truven Health Analytics, MI, USA]) from 1 January 2011 to 30 September 2016. This database includes fully de-identified information for approximately 46 million individuals in the USA. As such, this work does not meet the definition of research involving human participants and is not subject to regulation by the US Department of Health and Human Services' Protection of Human Subjects.

\section{Study design}

The index date was defined as the date of ASCT plus 90 days. Patients, who received a first-line treatment regimen for MM followed by ASCT, were assigned to one of two cohorts based on their maintenance treatment post-ASCT: Len-Mt or no maintenance therapy.

Treatment outcomes, including duration of maintenance, TTTD, TTNT and healthcare costs beyond the index date, were compared between patients who received Len-Mt ( $<20 \mathrm{mg} /$ day for $\geq 21$ of 28 days) versus those who received no maintenance therapy (Figure 1). The timing of receipt of therapy after ASCT and the number of cycles of treatment were examined to identify maintenance therapy. Patients were followed until death, end of study eligibility or data cutoff. 


\section{Study population}

The following inclusion criteria were applied: adults with $\geq 2 \mathrm{MM}$ diagnosis codes $\geq 30$ days apart during the 12 months prior to, and 3 months after their first observed MM diagnosis (the initial diagnosis date); receipt of a treatment of interest (bortezomib, carfilzomib, cyclophosphamide, dexamethasone, doxorubicin, doxorubicin liposomal, lenalidomide, melphalan, pomalidomide, thalidomide and vincristine); continuous enrollment for $\geq 6$ months before their initial diagnosis date (or initial induction therapy, if this was earlier) and $\geq 6$ months after their initial induction therapy; $\geq 1$ full cycle of MM treatment with a valid first-line regimen; and evidence of ASCT during the follow-up period. Supplementary Table 1 includes a full list of all diagnostic claims codes used.

Exclusion criteria were as follows: evidence of prior MM diagnosis or treatment (except dexamethasone or prednisone) or ASCT at any time prior to the observed MM diagnosis date; evidence of prior MM treatment (including ASCT) between the initial diagnosis date and initial induction therapy date; missing data (e.g., age and sex); $\geq 2$ identical claims for lymphomas and/or leukemias of interest (Supplementary Table 1 for codes) $\geq 30$ days apart after the initial induction therapy date; received tandem transplants (second transplant $<270$ days after initial transplant); or underwent ASCT within 60 days of initial induction therapy.

Patients assigned to the Len-Mt cohort received their first dose of post-ASCT lenalidomide monotherapy $<20 \mathrm{mg} /$ day for $\geq 21$ of 28 days (without steroids). Patients assigned to the no-maintenance cohort received no maintenance therapy (defined as no treatment with lenalidomide $<20 \mathrm{mg} /$ day for $\geq 21$ of 28 days, bortezomib once weekly for 4 weeks [days $1,8,15$ and 22 of a 35 day cycle] or thalidomide $50-200 \mathrm{mg} /$ day for $\geq 21$ of 28 days; either with or without steroids). Treatments not meeting these definitions were considered to be either consolidation therapy (if therapy was initiated $\leq 90$ days post-ASCT or there were one or two cycles during 90-270 days post-ASCT) or a next-line regimen. For consolidation therapy lasting one or two cycles, the next treatment was considered maintenance therapy; otherwise, it was considered next-line treatment. For consolidation therapy lasting $>2$ cycles and initiated within 90 days post-ASCT, next-line treatment was defined as the consolidation initiation date plus 60 days. Steroids alone and maintenance therapy were not considered to be next-line regimens.

\section{Outcomes}

Baseline characteristics and comorbidities were evaluated during the 365 days prior to the index date. Comorbidities within 90 days post-ASCT were also evaluated.

Duration of maintenance was measured from the initiation of lenalidomide maintenance to the last reported lenalidomide claim plus the day of supply. This was reported for patients receiving Len-Mt alone and was stratified by patient status as follows: (1) still receiving maintenance therapy (i.e., the maintenance end date was after the end of the study or the last administration/prescription would last beyond the end of the evaluation period); (2) progressed to next-line regimen during the eligible follow-up period; (3) in a treatment gap (i.e., finished maintenance but no next-line regimen was observed; (4) gap exists between maintenance end date and end of study $\leq 180$ days); or lost to follow-up (i.e., finished maintenance but no next-line regimen observed; gap exists between maintenance end date and end of study $>180$ days). Treatment was considered discontinued if patients were classified as being either (2) or (3) from the previous list. TTTD was defined as duration from initiation to discontinuation of lenalidomide maintenance. Patients classified as (1) or (4) from the previous list were censored at the last follow-up time.

TTNT, a disease progression proxy, was defined as the duration from the index date until the initiation of a subsequent line of treatment. This was compared between the Len-Mt and no-maintenance therapy cohorts.

Mean total healthcare costs (per patient per month [PPPM]) were compared between the two cohorts at 0-12, 12-24 and 24-36 months after the index date. Costs were inflated to September 2016 values using the Consumer Price Index US Medical Database and adjusted accordingly. Total healthcare costs included pharmacy costs (oral MM treatments; other oral medications); outpatient costs (intravenous MM and chemotherapy-related treatments; emergency department visits; outpatient physician visits, including ancillary, laboratory, radiology and surgery-related services); and inpatient costs. Claims codes are detailed in Supplementary Table 1.

\section{Statistical analysis}

Baseline characteristics were reported as means and standard deviations (SD) or percentages and number of patients. These were compared between cohorts using $\chi^{2}$ test or Fisher's exact test for categorical variables, and t test or Wilcoxon rank-sum test for continuous variables. 


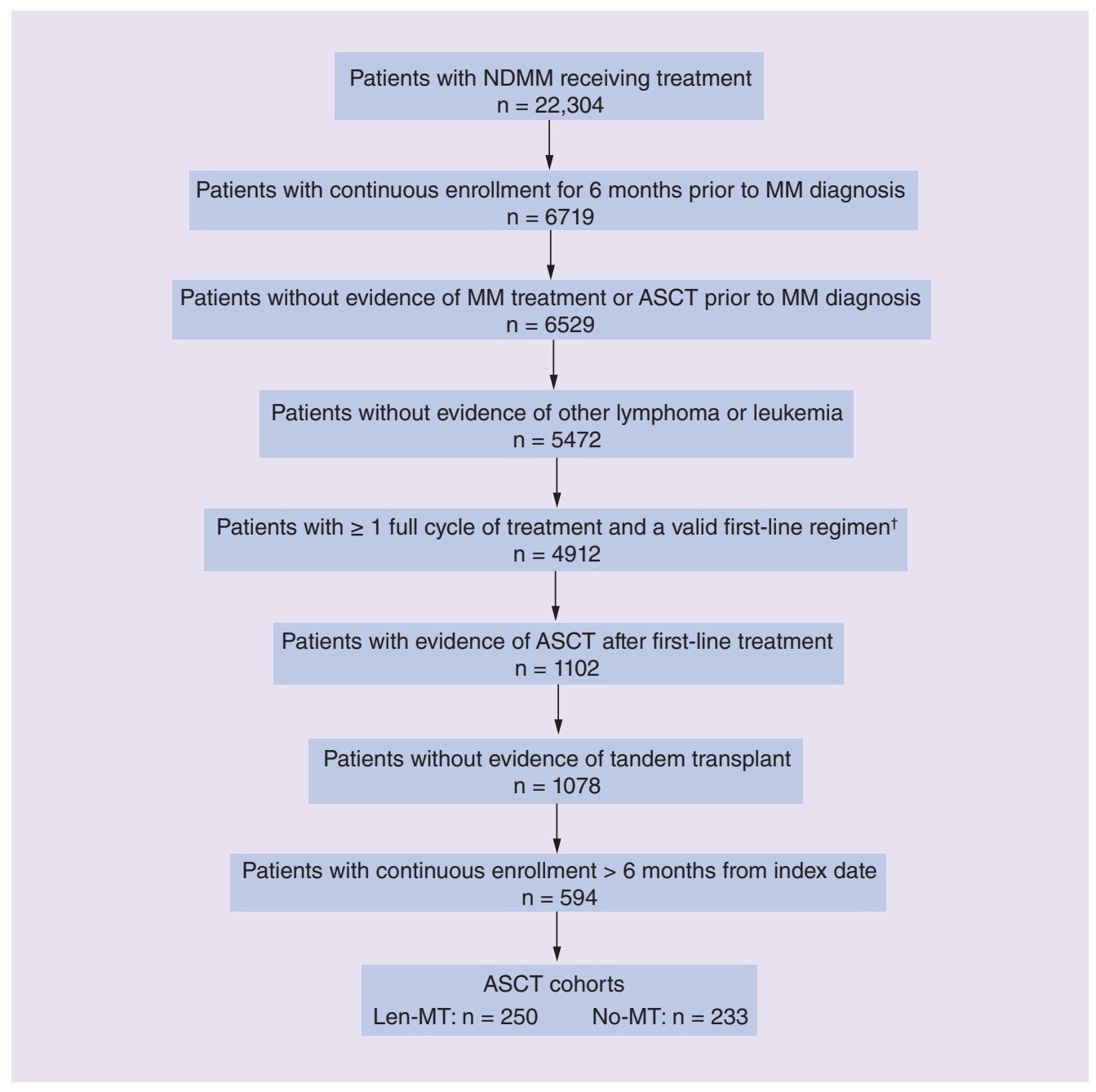

Figure 2. Study flow chart. ${ }^{\dagger}$ Valid first-line regimens were defined as bortezomib/dexamethasone $(n=1367)$, lenalidomide/dexamethasone $(n=1106)$, lenalidomide/bortezomib/dexamethasone $(n=963)$, cyclophosphamide/ bortezomib/dexamethasone $(n=552)$, steroid monotherapy $(n=479)$, other doublet $(n=307)$ and other $(n=138)$.

ASCT: Autologous stem cell transplantation; MM: Multiple myeloma; NDMM: Newly diagnosed multiple myeloma; No-Mt: No maintenance therapy; Len-Mt: Lenalidomide-only maintenance.

Median TTTD was calculated for the Len-Mt cohort based on the Kaplan-Meier method. Kaplan-Meier and Cox proportional hazards models were performed to evaluate TTNT for both cohorts. Unadjusted p values were based on log-rank tests. Adjusted p values and HRs were based on Cox proportional hazard models, with sex, region, pulmonary embolism and pneumonia (all at baseline [i.e., within 365 days before index date]), and thrombocytopenia (within 90 days post-ASCT) as covariates.

Generalized linear regression models with negative binomial distribution and log link function were used to assess differences in costs, unadjusted and adjusted, as previously detailed.

\section{Results}

\section{Study population}

Of the 69,950 patients with a recorded MM diagnosis during the study period (from 1 January 2011 to 30 September 2016), 594 met the inclusion criteria (Figure 2). Of these, 297 (50.0\%) received Len-Mt therapy, 233 $(39.2 \%)$ received no maintenance therapy and $64(10.8 \%)$ received another maintenance therapy (lenalidomide with steroids; bortezomib with or without steroids; or thalidomide with or without steroids). Of the 297 patients 
Table 1. Baseline ${ }^{\dagger}$ demographics and clinical characteristics and comorbidities within 90 days post-autologous hematopoietic stem cell transplantation.

\begin{tabular}{|c|c|c|c|}
\hline Variable & Len-Mt $(n=250)$ & No maintenance $(n=233)$ & p-value \\
\hline \multicolumn{4}{|l|}{ Baseline } \\
\hline Age, mean \pm SD (years) & $58.5 \pm 8.4$ & $58.0 \pm 8.5$ & 0.52 \\
\hline Male & $130(52.0)$ & $143(61.4)$ & 0.038 \\
\hline US geographic region: & & & 0.0003 \\
\hline - South & $129(51.6)$ & $78(33.5)$ & \\
\hline - North central & $55(22.0)$ & $58(24.9)$ & \\
\hline - Northeast & $42(16.8)$ & $48(20.6)$ & \\
\hline - West & $22(8.8)$ & $46(19.7)$ & \\
\hline - Unknown & $2(0.8)$ & $3(1.3)$ & \\
\hline Health plan type: & & & 0.40 \\
\hline - Health maintenance organization & $25(10.0)$ & $20(8.6)$ & \\
\hline - Preferred provider organization & $151(60.4)$ & $138(59.2)$ & \\
\hline - Point of service/other & $51(20.4)$ & $60(25.8)$ & \\
\hline - Comprehensive & $23(9.2)$ & $15(6.4)$ & \\
\hline Payer type: & & & 0.66 \\
\hline - Commercial & $190(76.0)$ & $181(77.7)$ & \\
\hline - Medicare & $60(24.0)$ & $52(22.3)$ & \\
\hline \multicolumn{4}{|l|}{ Comorbidities $\ddagger$ : } \\
\hline - Liver, gallbladder or pancreatic disease & $47(18.8)$ & $23(9.9)$ & 0.0053 \\
\hline Charlson Comorbidity Index, mean \pm SD & $5.2 \pm 3.2$ & $5.3 \pm 3.4$ & 0.52 \\
\hline Charlson Comorbidity Index group: & & & 0.51 \\
\hline-0 & $70(28.0)$ & $55(23.6)$ & \\
\hline$-1-2$ & $74(29.6)$ & $70(30.0)$ & \\
\hline$-3-4$ & $106(42.4)$ & $108(46.4)$ & \\
\hline \multicolumn{4}{|l|}{ MM medical events of interest ${ }^{\S}$ : } \\
\hline - Pneumonia & $31(12.4)$ & $46(19.7)$ & 0.028 \\
\hline - Pulmonary embolism & $6(2.4)$ & $14(6)$ & 0.047 \\
\hline \multicolumn{4}{|l|}{ Comorbidities within 90 days post-ASCT: } \\
\hline - Anemia & $138(55.2)$ & $144(61.8)$ & 0.14 \\
\hline - Neutropenia & $87(34.8)$ & $92(39.5)$ & 0.29 \\
\hline - Thrombocytopenia & $39(15.6)$ & $58(24.9)$ & 0.011 \\
\hline - Sleep disorders & $16(6.4)$ & $18(7.7)$ & 0.57 \\
\hline - Renal disease excluding renal failure & $12(4.8)$ & $14(6.0)$ & 0.56 \\
\hline - Renal failure/dialysis & $9(3.6)$ & $9(3.9)$ & 0.88 \\
\hline - Liver, gallbladder or pancreatic disease & $10(4.0)$ & $3(1.3)$ & 0.066 \\
\hline \multicolumn{4}{|c|}{$\begin{array}{l}\text { Data presented as } n(\%), \text { unless otherwise stated. } \\
\text { †During the } 365 \text { days prior to the index date. } \\
\text { ¥A total of } 14 \text { comorbidities were assessed; only those with a statistically significant difference are shown. } \\
\text { § total of } 19 \text { MM medical events of interest were assessed; only those with a statistically significant difference are shown. } \\
\text { ASCT: Autologous stem cell transplantation; Len-Mt: Lenalidomide-only maintenance; MM: Multiple myeloma; SD: Standard }\end{array}$} \\
\hline
\end{tabular}

who received lenalidomide only, 47 received lenalidomide before the index date and were excluded from the present study; 250 of the 297 (84.2\%) patients who received Len-Mt after the index date were included in the study.

The two cohorts were similar in terms of age and payer type, but there were significantly fewer men in the LenMt therapy cohort $(\mathrm{p}=0.038)$ and significant variations were observed in the geographic distribution of patients (Table 1). Although baseline Charlson Comorbidity Index score was similar in both cohorts, liver, gallbladder or pancreatic disease was significantly more prevalent in the Len-Mt cohort ( $p=0.0053$; Table 1). MM medical events of interest at baseline were generally similar between the two cohorts, although patients in the Len-Mt cohort were significantly less likely to have pneumonia or pulmonary embolism $(\mathrm{p}=0.028$ and $\mathrm{p}=0.047$, respectively; Table 1$)$. In the 90-day period post-ASCT, the prevalence of liver, gallbladder or pancreatic disease had decreased and was no 


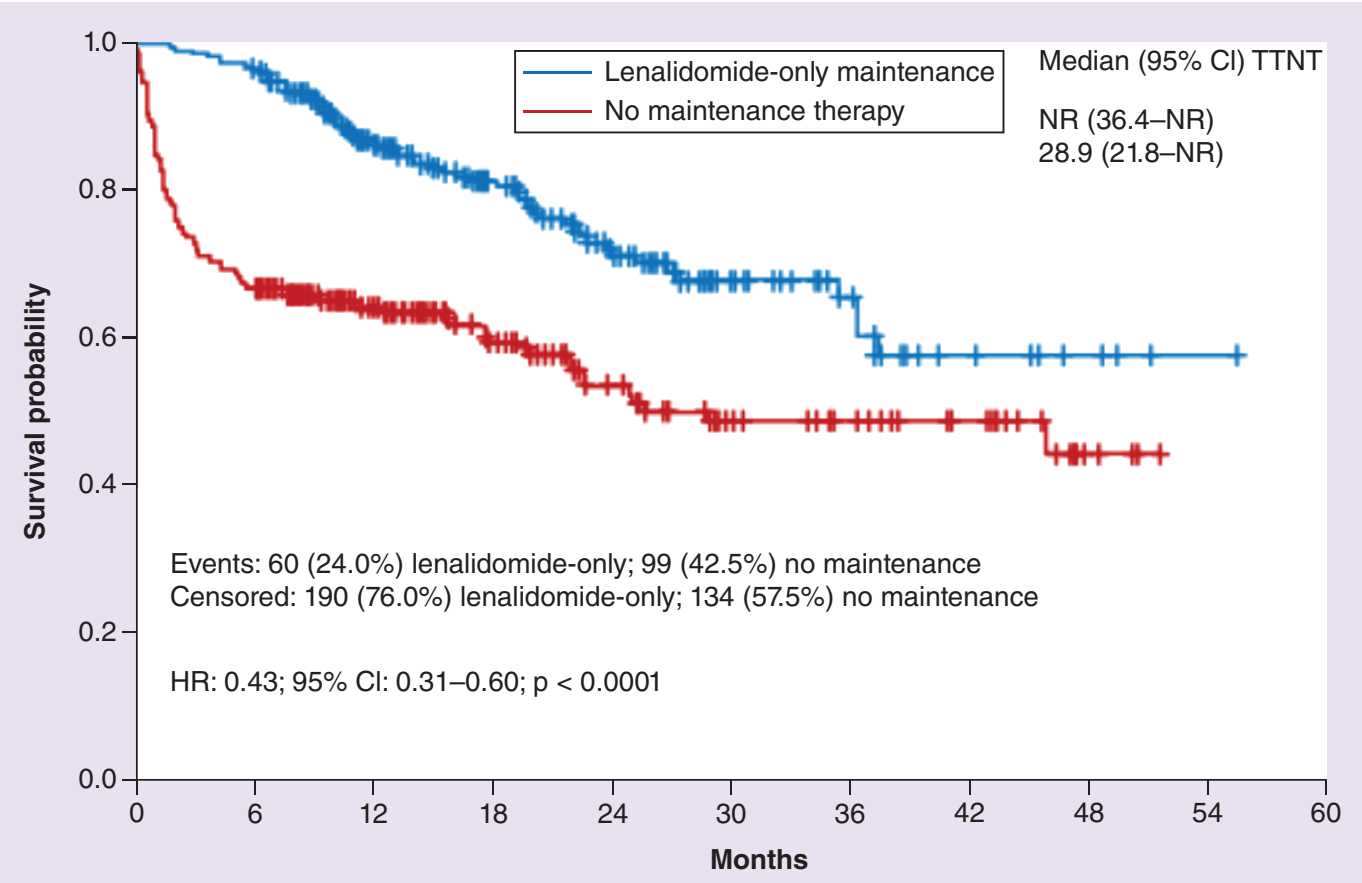

Patients at risk

$\begin{array}{ccccccccccc}\begin{array}{c}\text { Lenalidomide-only } \\ \text { maintenance }\end{array} & 251 & 165 & 121 & 74 & 42 & 26 & 15 & 5 & 1 & 0 \\ \text { No maintenance } & 233 & 156 & 103 & 70 & 47 & 31 & 24 & 17 & 4 & 0\end{array}$

Figure 3. Kaplan-Meier graph of time to next treatment.

HR: Hazard ratio; NR: Not reached; TTNT: Time to next treatment.

longer significantly different between the two cohorts; however, significantly fewer patients in the Len-Mt cohort had thrombocytopenia than those assigned to the no-maintenance cohort $(\mathrm{p}=0.011$; Table 1$)$.

Mean \pm SD duration from the start of the first-line regimen to ASCT $(5.48 \pm 1.4$ months vs $5.47 \pm 1.5$ months; $\mathrm{p}=0.9589)$ and from the index date to the end of follow-up $(22.1 \pm 11.8$ months vs $21.0 \pm 12.5$ months; $\mathrm{p}=0.14$ ) was similar in the Len-Mt and no-maintenance therapy cohorts.

Duration of maintenance \& time to treatment discontinuation

Median duration of maintenance therapy among all patients receiving Len-Mt therapy was 10.0 months. Median TTTD using the Kaplan-Meier method was 21.02 months (95\% CI: 16.8-25.8; data not shown). At the end of the follow-up period, 96 patients receiving Len-Mt (38.4\%) were still on maintenance therapy, $60(24.0 \%)$ had initiated a next-line regimen, $51(20.4 \%)$ were in a treatment gap and $43(17.2 \%)$ had been lost to follow-up. Median duration of Len-Mt for each group was 15.5 months (still on maintenance), 7.5 months (initiated next line), 4.8 months (treatment gap) and 13.0 months (lost to follow-up).

\section{Time to next treatment}

Patients in the Len-Mt cohort were less likely initiate a next-line treatment compared with those in the no-maintenance cohort $(24.0$ vs $42.5 \%)$ and had significant longer TTNT (HR: 0.43 ; $95 \%$ CI: $0.31-0.60 ; \mathrm{p}<0.0001$; Figure 3). The probability of receiving next-line treatment was lower in the Len-Mt cohort compared with the no-maintenance cohort at each time point (12 months: 13.9 vs $39.2 \%$; 24 months: 28.4 vs $45.2 \%$; 36 months: 33.9 vs $53.8 \%)$.

\section{Healthcare resource use \& costs}

Total adjusted healthcare costs were higher in the Len-Mt cohort than in the no-maintenance therapy cohort during months $0-12,12-24$ and 24-36 (Figure 4). For example, during months $0-12$, the mean costs in US dollars (USD) 


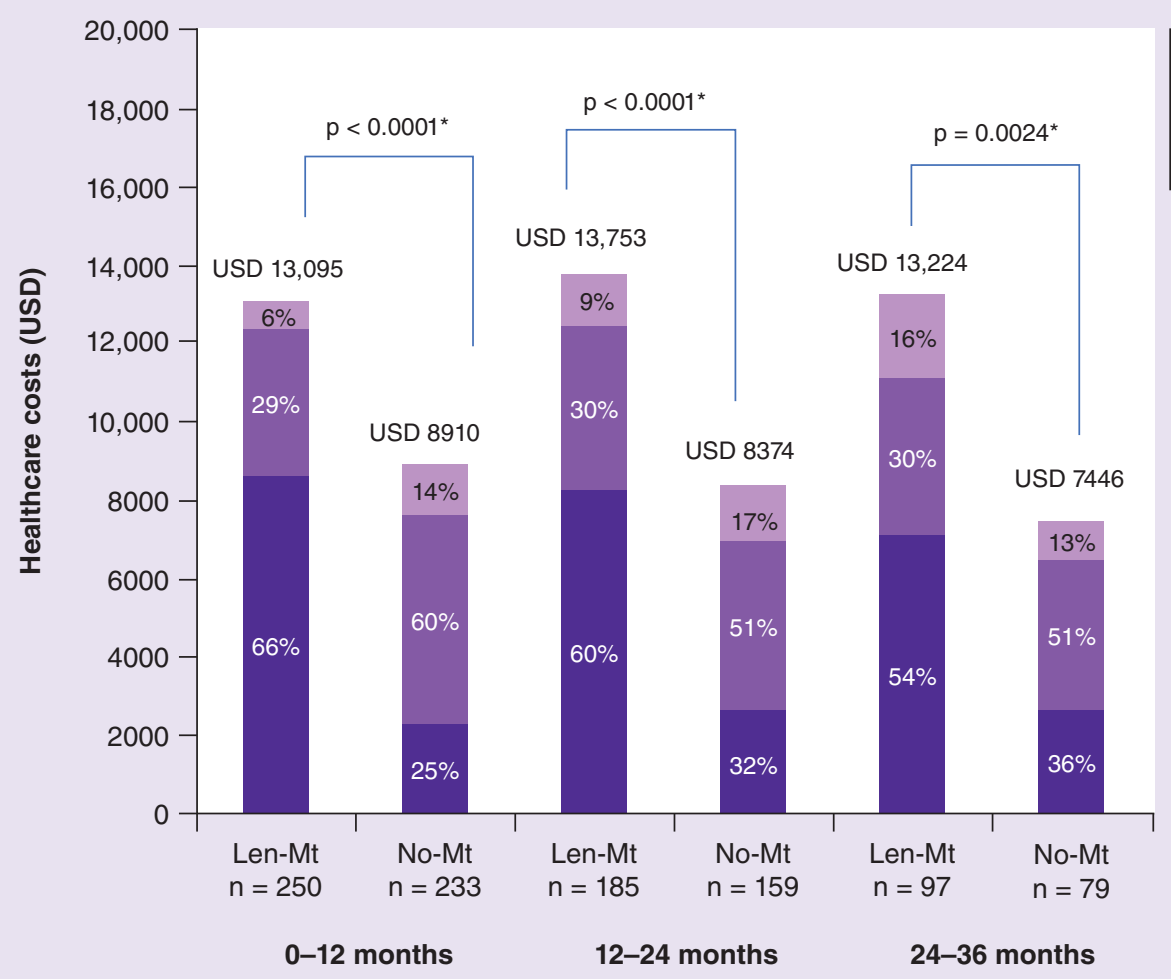

Figure 4. Mean monthly healthcare costs and differences (lenalidomide-only vs no maintenance) during 0-12, 12-24 and 24-36 months postindex date. Data are mean \pm SD costs per patient per month (USD). Percentages are rounded to the nearest unit.

*Calculated from generalized linear regression analysis with negative binomial distribution and log link function; for multivariate analysis, sex, region, thrombocytopenia condition, pulmonary embolism and pneumonia were included as covariates.

No-Mt: No maintenance; Len-Mt: Lenalidomide-only maintenance; SD: Standard deviation.

were USD 13,095 in the Len-Mt cohort and USD 8910 in the no-maintenance cohort (adjusted difference +USD 4514; $\mathrm{p}<0.0001$ ). However, individual monthly patient costs varied widely during all three periods. In addition, any costs associated with disease progression were captured in total healthcare costs.

The increased cost in the Len-Mt cohort was largely due to higher mean pharmacy costs. On average, patients in the Len-Mt cohort had three pharmacy claims each month compared with two claims in the no-maintenance cohort (Supplementary Table 2). The pharmacy costs were mainly attributable to oral MM treatments. Pharmacy costs in the no-maintenance cohort were attributable to steroids, thalidomide, bortezomib and lenalidomide among others. These regimens were not considered maintenance treatment and were classed as next-line therapies; a total of 99 patients in the no-maintenance cohort (42.5\%) progressed to next-line therapies. However, patients in both cohorts had fewer pharmacy claims for MM versus other oral medications (Supplementary Table 2).

Outpatient costs were significantly lower in the Len-Mt versus no-maintenance cohort during months $0-12$ (USD 3761 vs USD 5360; adjusted difference USD 1708; $<<0.0001$ ), mainly due to lower intravenous MM and chemotherapy-related costs (Figure 4) and significantly lower pharmacy usage ( $<<0.0001$; Supplementary Table 2). However, during months 12-24 and 24-36, outpatient costs and usage (Supplementary Table 2) were similar in both cohorts. During months $0-12$, most of the outpatient costs in both cohorts were due to physician visits or ancillary, laboratory, radiology and surgery-related services, whereas intravenous MM treatments and chemotherapy-related treatment comprised a larger proportion of outpatient costs during months 24-36 (Figure 4). However, more claims were made for physician visits and ancillary, laboratory, radiology and surgery-related services than intravenous MM treatments and chemotherapy-related regimens in both cohorts during all three periods (Supplementary Table 2). Emergency department costs accounted for a very small proportion of overall outpatient costs. 
Inpatient usages (Supplementary Table 2) were not significantly different between the two cohorts during all three periods.

\section{Discussion}

In this retrospective observational study, patients with NDMM who received Len-Mt after ASCT achieved significantly longer TTNT (a proxy for disease progression) than those who received no maintenance therapy. Higher pharmacy costs observed with Len-Mt (vs no maintenance as expected) were offset by significant reductions in outpatient costs beyond 1 year.

This analysis revealed some compelling differences in demographic characteristics observed between patients prescribed Len-Mt therapy compared with those who were not. More women received Len-Mt than no maintenance ( 48.0 vs $38.6 \%$, respectively; $\mathrm{p}=0.038$ ). Differences between geographic regions were also present. In the south, more patients received Len-Mt than not (51.6 vs 33.5\%, respectively), whereas this trend was reversed in the west (8.8 vs $19.7 \%$, respectively). No significant differences were observed in Len-Mt use by health plan or payer type.

Median duration of Len-Mt was 10 months, whereas median TTTD was 21 months, as calculated by KaplanMeier. This is somewhat shorter than the median durations of Len-Mt reported in three RCTs ( $\sim 2-3$ years) [8,9,13,18], and the Connect MM Registry, which reported durations of 27.3 months [19] and 35.2 months, respectively [20]. This difference may be explained by the fact that the present analysis was conducted using administrative claims data rather than registry data, as was done with the Connect MM Registry analyses $[19,20]$. Rules to determine treatment discontinuation may also differ. Within registries, physicians may utilize breaks in maintenance therapy, which are reflected in a registry database but not observed in claims datasets. Moreover, the duration of follow-up was longer within the Connect MM Registry (39.3 months) $[19,20]$ than the present administrative claims database study (22.1 months). The shorter median duration of Len-Mt and TTTD may reflect the patients in the maintenance therapy cohort who were still receiving maintenance therapy after the end of the follow-up period.

Whether lenalidomide maintenance therapy should be used continuously or for a fixed period has been previously highlighted [18]. Two retrospective analyses reported that longer durations of lenalidomide maintenance therapy after ASCT were associated with significant improvements in PFS and OS [21,22], leading those study authors to suggest that lenalidomide maintenance therapy should be continued for as long as it is tolerated or until disease progression. Richardson et al. [18] also advocated the use of lenalidomide maintenance therapy after ASCT until progression as standard of care for patients with NDMM, despite the increased risk of second primary malignancies [8-10,14,23], because the net health benefits are significant.

The TTNT was significantly longer with Len-Mt versus no maintenance therapy (HR: 0.43; 95\% CI: $0.31-$ $0.60 ; \mathrm{p}<0.0001)$. This real-world finding supports data from various RCTs, which have shown that lenalidomide maintenance therapy has a positive impact on disease progression $[8,9,12,13]$.

Data from the Connect MM Registry also suggest that Len-Mt therapy significantly improves PFS versus no maintenance therapy $[19,20,24]$.

Len-Mt therapy was associated with additional adjusted pharmacy costs (approximately USD 6000 PPPM) in the current study. This finding is consistent with results from LeBlanc et al. [25], who conducted a cost analysis from the perspective of the Canadian healthcare system in 2014 using clinical inputs from published trials. They reported average costs of Canadian dollars (CAD) 131,765/year ( USD 8700/month using March 2018 conversion values) for lenalidomide maintenance therapy after ASCT in Canada (based on drug, administration, and pharmacy costs alone). They found that incorporating costs for treating adverse events, dose adjustments and the treatment of second primary malignancies reduced this to CAD 108,085/year ( USD 7100/month), primarily due to cost-savings associated with dose reductions [25].

Two US studies examined the costs of lenalidomide maintenance therapy in patients with transplantineligible NDMM [26,27]. Garrison et al. [26] used a Markov model and data from RCTs to estimate lifetime costs from the US payer perspective. Data from three trials were used comparing melphalan/prednisone and melphalan/prednisone/bortezomib (VISTA) [28], melphalan/prednisone/thalidomide (IFM 99-06) [29] and melphalan/prednisone/lenalidomide, with or without lenalidomide maintenance therapy (interim analysis of MM015) [30]. The total cost of lenalidomide maintenance therapy from this analysis was USD 248,358 [26]. However, cost, life year and quality-adjusted life year outcomes for melphalan/prednisone/lenalidomide without maintenance therapy were not reported, so the effect of adding lenalidomide maintenance to melphalan/prednisone/lenalidomide was not examined. In a later modeling study, Kim et al. [27] used published MM-015 data [31] to estimate cost outcomes for all three arms of the study. Although the addition of lenalidomide maintenance was estimated to 
increase the 'average cumulative cost per patient' by 1.8 -fold compared with melphalan/prednisone/lenalidomide and no maintenance therapy (USD 309,173 vs USD 167,862 [2011 USD]), the average cumulative cost per progression-free survivor' was reduced by $55.6 \%$ (USD 690,111 vs USD 1,555,443).

Fonseca et al. [3] examined trends in the overall costs of treating patients with NDMM during 2000-2014. Using data from the Truven Health Analytics MarketScan Commercial and Medicare Supplemental Databases, they reported that total all-cause healthcare costs steadily increased during this time (from USD 3263 PPPM in 2000 to USD 14,656 PPPM in 2014). Costs were mainly due to outpatient services (60\% in 2000; 49\% in 2014), followed by hospitalizations (22 and 33\%, respectively), and MM treatment-related drug costs (11 and 29\%, respectively). Therefore, although MM treatment-related drug costs considerably increased during the study (from USD 346 PPPM in 2000 to USD 4179 PPPM in 2014), they contributed less to the overall cost of healthcare among those with NDMM than the total contributions of outpatient services and hospitalizations during the time studied. In the present study, the overall cost was higher for patients receiving Len-Mt therapy, but this was mainly due to increased pharmacy costs, with outpatient costs either lower than or similar to the no-maintenance cohort and inpatient costs similar between both groups. It is important to note that the increased costs in the Len-Mt group were associated with extended TTNT, reflecting reduced likelihood of initiating a new therapy due to progression.

A real-world analysis of medical records from five European countries (France, Germany, Italy, Spain and the UK) showed that patients receiving lenalidomide maintenance therapy had longer time to disease progression (39 vs 30 months) and considerably lower mean PPPM costs compared with patients who did not (EUR 638 vs EUR 1001), reflecting lower costs for hospitalizations, healthcare professional visits and monitoring tests, although costs for supportive drugs and other treatments were higher in those receiving maintenance therapy [32]. Notably, this study also showed that $7 \%$ of patients with MM received maintenance therapy after ASCT, and, of those, $88 \%$ received lenalidomide monotherapy as maintenance. Because lenalidomide has been established as an effective maintenance treatment after ASCT [8,9,12,13] and in patients who are ineligible for ASCT [31], several lenalidomidebased maintenance regimens are now under investigation (as reviewed by Richardson et al. [18]). Adding additional treatments to lenalidomide maintenance therapy may increase its efficacy but doing so will also incur additional costs and potentially increase the rate of toxicity. Therefore, further research into the costs of lenalidomide maintenance therapy (with or without additional therapies) is warranted.

\section{Limitations}

Although the present study provides information on actual costs among real-world patients with NDMM who have undergone ASCT, its retrospective, observational nature is a limitation. Many patients with MM who received treatment were not included in the study due to lack of continuous enrollment for 6 months before their MM diagnosis and after their index date. The requirement for evidence of ASCT after first-line treatment also resulted in a reduction of the number of patients who were eligible for inclusion in this study (Figure 2). It should also be noted that a third of patients who were included in the present study contributed to the cost data for months 24-36.

Any economic inferences made from claims records depend on the accuracy of coding. Because the reasons for receiving treatment are not included in the claims database, it is possible that some patients may have been incorrectly classified as receiving Len-Mt or no maintenance therapy. Factors that affect clinical care are not available in the claims database, and proxies were used to indicate disease progression (e.g., changes in therapy). Therefore, the present TTNT results are not necessarily comparable with PFS data reported in RCTs. However, it is likely that the TTNT data presented here reflects RCT outcomes in terms of PFS. Moreover, claims database analyses represent paid claims and do not include out-of-pocket costs incurred by patients. As these would vary depending on an individual patient's insurance benefit (i.e., deductible, coinsurance vs copay structure) and would not include other expenses such as those associated with travel to receive treatment, these analyses may not necessarily represent the complete cost of treatment.

Although patients from across the USA were included, these results may not be generalizable to the entire US population. Furthermore, only patients with commercial health insurance or Medicare coverage who were included in the Truven MarketScan database were included. Because no patients covered by Medicaid were included, our results may not be applicable to this population. Given the differences in healthcare systems across the world, these results may not be applicable to other countries. 


\section{Conclusion}

Using a US claims database, this study examined real-world data on Len-Mt therapy to understand its effectiveness outside the RCT setting. Patients with NDMM who received Len-Mt treatment remained on therapy for $\geq 1$ year after ASCT and had significantly prolonged TTNT compared with those who received no maintenance therapy. They were also less likely to experience disease progression within the study period. This improvement in outcome was offset by significantly higher overall costs, mainly due to increased pharmacy costs. However, patients receiving Len-Mt therapy had significantly lower outpatient costs during the first year after ASCT compared with those who did not receive maintenance therapy. The higher outpatient costs in the no-maintenance therapy cohort may be driven by the need for next-line regimens in a higher proportion of patients. Additional follow-up is required to more accurately assess the cost of Len-Mt treatment over a prolonged period of time.

\section{Summary points}

- Treatment options for patients with newly diagnosed multiple myeloma include: primary therapy with multidrug regimens, autologous stem cell transplantation for eligible patients and maintenance therapy.

- The clinical benefits of maintenance therapy after autologous stem cell transplantation are clear, but real-world evidence is lacking.

- This claims database study showed that patients receiving lenalidomide-only maintenance therapy remained on therapy for > 1 year (median 15.5 months) had a longer time to next treatment, and were less likely than patients receiving no maintenance therapy to experience disease progression within the study time period.

- The significant improvement in outcomes seen with lenalidomide-only maintenance therapy was associated with increases in overall and pharmacy costs during months 0-12, 12-24 and 24-36, but outpatient costs were lower during months $0-12$ when compared with patients receiving no maintenance therapy.

- Additional follow-up is required to assess total healthcare costs in subsequent years.

Supplementary data

To view the supplementary data that accompany this paper please visit the journal website at: www.futuremedicine.com/doi/sup $\mathrm{pl} / 10.2217 /$ fon-2019-0422

Author contributions

All authors contributed to the concept and design of the study; all authors contributed to the acquisition, analysis and interpretation of the data for the study; all authors contributed to the drafting of the manuscript, revised the manuscript critically for important intellectual content and approved the final version to be published.

\section{Acknowledgments}

The authors would like to thank Q Ni, an employee of Celgene Corporation at the time of the study, for her valuable contribution to data analysis and interpretation.

\section{Disclaimer}

The study sponsor was involved in the study design; collection, analysis and interpretation of data; writing of the manuscript; and the decision to submit the manuscript. The authors are fully responsible for all content and editorial decisions for this manuscript.

Financial \& competing interest disclosure

P Hari reports consultancy, research funding and honoraria from Celgene Corporation. B Ung reports fellowship funded by Celgene Corporation. S Abouzaid, A Agarwal and K Parikh report employment with and equity ownership in Celgene Corporation. This study was funded by Celgene Corporation (NJ, USA). The authors have no other relevant affiliations or financial involvement with any organization or entity with a financial interest in or financial conflict with the subject matter or materials discussed in the manuscript apart from those disclosed.

The authors received writing and editorial assistance in the preparation of this manuscript from R Morland, PhD, of Excerpta Medica, funded by Celgene Corporation.

Open access

This work is licensed under the Attribution-NonCommercial-NoDerivatives 4.0 Unported License. To view a copy of this license, visit http://creativecommons.org/licenses/by-nc-nd/4.0/ 


\section{References}

Papers of special note have been highlighted as: $\bullet$ of interest; $\bullet \bullet$ of considerable interest

1. Siegel RL, Miller KD, Jemal A. Cancer statistics, 2019. CA Cancer J. Clin. 67(1), 7-34 (2019).

2. Costa LJ, Brill IK, Omel J, Godby K, Kumar SK, Brown EE. Recent trends in multiple myeloma incidence and survival by age, race, and ethnicity in the United States. Blood Adv. 1(4), 282-287 (2017).

3. Fonseca R, Abouzaid S, Bonafede M et al. Trends in overall survival and costs of multiple myeloma, 2000-2014. Leukemia 31(9), 1915-1921 (2017).

4. National Comprehensive Cancer Network. Multiple myeloma, version 3.2019 (2019). www.nccn.org/professionals/physician_gls/pdf/myeloma.pdf

- The NCCN guidelines report the sequential management decisions and interventions for the treatment of multiple myeloma (MM) in the US.

5. Moreau P, San Miguel J, Sonneveld P et al. Multiple myeloma: ESMO clinical practice guidelines for diagnosis, treatment and follow-up. Ann. Oncol. 28(Suppl. 4), iv52-iv61 (2017).

6. US FDA. Lenalidomide (revlimid) (2017). www.fda.gov/drugs/informationondrugs/approveddrugs/ucm542791.htm

7. European Medicines Agency. Revlimid (lenalidomide) (2017). www.ema.europa.eu/docs/en_GB/document_library/Summary_of_opinion/human/000717/WC500220225.pdf

8. Attal M, Lauwers-Cances V, Marit G et al. Lenalidomide maintenance after stem-cell transplantation for multiple myeloma. N. Engl. J. Med. 366(19), 1782-1791 (2012).

- Pivotal Phase III trial showing the survival benefits of lenalidomide maintenance versus placebo after autologous hematopoietic stem cell transplantation (ASCT) for patients with newly diagnosed multiple myeloma (NDMM).

9. McCarthy PL, Owzar K, Hofmeister CC et al. Lenalidomide after stem-cell transplantation for multiple myeloma. N. Engl. J. Med. 366(19), 1770-1781 (2012).

- Pivotal Phase III trial showing that lenalidomide maintenance after ASCT prolongs the time to disease progression compared with placebo in patients with NDMM.

10. Holstein SA, Jung SH, Richardson PG et al. Updated analysis of CALGB (Alliance) 100104 assessing lenalidomide versus placebo maintenance after single autologous stem-cell transplantation for multiple myeloma: a randomised, double-blind, Phase III trial. Lancet Haematol. 4(9), e431-e442 (2017).

11. McCarthy PL, Richardson P, Suman V et al. Survival analysis from the CALGB study of lenalidomide maintenance therapy in newly diagnosed multiple myeloma post-autologous stem cell transplantation adjusted for crossover (Alliance 100104). Blood 132, 4737 (2018).

12. Jackson GH, Davies F, Pawlyn C et al. Lenalidomide induction and maintenance therapy for transplant eligible myeloma patients: results of the Myeloma XI study. J. Clin. Oncol. 35(Suppl.15), 8009 (2017).

13. Palumbo A, Cavallo F, Gay F et al. Autologous transplantation and maintenance therapy in multiple myeloma. N. Engl. J. Med. 371(10), 895-905 (2014).

14. McCarthy PL, Holstein SA, Petrucci MT et al. Lenalidomide maintenance after autologous stem-cell transplantation in newly diagnosed multiple myeloma: a meta-analysis. J. Clin. Oncol. 35(29), 3279-3289 (2017).

-. This meta-analysis, which includes three randomized clinical trials (CALGB 100104, GIMEMA RV-MM-PI-209 and IFM 2005-02), demonstrates significant survival benefits with lenalidomide maintenance after ASCT for patients with NDMM compared with placebo.

15. Arikian SR. Patterns of total cost and economic consequences of progression for patients with newly diagnosed multiple myeloma. Curn. Med. Res. Opin. 31(6), 1105-1115 (2015).

16. MacEwan JP, Batt K, Yin W et al. Economic impact of disease progression in Medicare patients with relapsed multiple myeloma. Blood 126(23), 2116 (2015).

17. Hagiwara M, Delea T, Panjabi S, Yucel E, Fonseca R. Burden of disease progression in patients with multiple myeloma who have received at least one line of therapy in the US. Blood 132(Suppl.1), 4754 (2018).

18. Richardson PG, Holstein SA, Schlossman RL, Anderson KC, Attal M, McCarthy PL. Lenalidomide in combination or alone as maintenance therapy following autologous stem cell transplant in patients with multiple myeloma: a review of options for and against. Expert Opin. Pharmacother. 18(18), 1975-1985 (2017).

-• Summarizes the efficacy and safety data of lenalidomide maintenance after ASCT in patients with NDMM.

19. Rifkin RM, Jagannath S, Durie BGM et al. Treatment outcomes and health care resource utilization in patients with newly diagnosed multiple myeloma receiving lenalidomide-only maintenance, any maintenance, or no maintenance: results from the Connect MM Registry. Clin. Ther. 40(7), 1193-1202 (2018).

20. Jagannath S, Abonour R, Durie BGM et al. Assessment of the impact of post-autologous stem cell transplant maintenance therapy on survival outcomes in patients with newly diagnosed multiple myeloma in the community-based Connect MM registry. Haematologica 102(Suppl.2), 507 (2017). 
21. Mian I, Milton DR, Shah N et al. Prolonged survival with a longer duration of maintenance lenalidomide after autologous hematopoietic stem cell transplantation for multiple myeloma. Cancer 122(24), 3831-3837 (2016).

22. Amsler IG, Jeker B, Mansouri Taleghani B et al. Prolonged survival with increasing duration of lenalidomide maintenance after autologous transplant for multiple myeloma. Leuk. Lymphoma 60(2), 511-514 (2019).

23. Jones JR, Cairns DA, Gregory WM et al. Second malignancies in the context of lenalidomide treatment: an analysis of 2732 myeloma patients enrolled to the Myeloma XI trial. Blood Cancer J. 6(12), e506 (2016).

24. Rifkin RM, Jagannath S, Durie BGM et al. Analysis of the Connect MM registry: treatment outcomes and healthcare resource utilization in patients with newly diagnosed multiple myeloma who received lenalidomide maintenance or no maintenance. Haematologica 102(Suppl. 2), 523-524 (2017).

25. LeBlanc R, Hollmann S, Tay J. Canadian cost analysis comparing maintenance therapy with bortezomib versus lenalidomide for patients with multiple myeloma post autologous stem cell transplant. J. Popul. Ther. Clin. Pharmacol. 23(1), e103-e113 (2016).

26. Garrison LP Jr, Wang ST, Huang H et al. The cost-effectiveness of initial treatment of multiple myeloma in the U.S. with bortezomib plus melphalan and prednisone versus thalidomide plus melphalan and prednisone or lenalidomide plus melphalan and prednisone with continuous lenalidomide maintenance treatment. Oncologist 18(1), 27-36 (2013).

27. Kim MY, Sposto R, Swaika A et al. Pharmacoeconomic implications of lenalidomide maintenance therapy in multiple myeloma. Oncology 87(4), 224-231 (2014).

28. San Miguel JF, Schlag R, Khuageva NK et al. Bortezomib plus melphalan and prednisone for initial treatment of multiple myeloma. $N$. Engl. J. Med. 359(9), 906-917 (2008).

29. Facon T, Mary JY, Hulin C et al. Melphalan and prednisone plus thalidomide versus melphalan and prednisone alone or reduced-intensity autologous stem cell transplantation in elderly patients with multiple myeloma (IFM 99-06): a randomised trial. Lancet 370(9594), 1209-1218 (2007).

30. Palumbo A, Dimopoulos MA, Delforge M et al. A Phase III study to determine the efficacy and safety of lenalidomide in combination with melphalan and prednisone (MPR) in elderly patients with newly diagnosed multiple myeloma. Blood 114(22), 613 (2009).

31. Palumbo A, Hajek R, Delforge $\mathrm{M}$ et al. Continuous lenalidomide treatment for newly diagnosed multiple myeloma. $N$. Engl. J. Med. 366(19), 1759-1769 (2012).

32. Ashcroft J, Judge D, Dhanasiri S, Taylor-Stokes G, Middleton C. Chart review across EU5 in MM post-ASCT patients. Int. J. Hematol. Oncol. 7(1), 05 (2018).

- This analysis highlights the health benefits and describes the costs associated with maintenance therapy after ASCT in European patients with MM. 